\title{
An Improved Finite Temperature Lanczos Method and Its Application to the Spin-1/2 Heisenberg Model on the Kagome Lattice
}

\author{
Tomo Munehisa \\ Faculty of Engineering, University of Yamanashi, Kofu, Japan \\ Email: munehisa@yamanashi.ac.jp
}

Received 1 May 2014; revised 9 June 2014; accepted 30 June 2014

Copyright (C) 2014 by author and Scientific Research Publishing Inc.

This work is licensed under the Creative Commons Attribution International License (CC BY).

http://creativecommons.org/licenses/by/4.0/

(c) (i) Open Access

\section{Abstract}

We present an improvement of the finite temperature Lanczos method in order to apply this method to systems at very low temperature. One proposal is to introduce two steps in this method. In the first step, we use the Chebyshev polynomial expansion to calculate $\exp \left(-\hat{H} / T_{1}\right) \mid$ random vector $\rangle$ at moderate temperature $T_{1}$. In the second step, we apply the ordinary finite temperature Lanczos method using the calculated state as the initial state of the Lanczos method. Another proposal is to employ a sampling method for selecting a random vector. By this sampling, we can improve an efficiency of calculations. Using the improved finite temperature Lanczos method, we calculate the specific heat of the spin-1/2 Heisenberg model on the kagome lattices of 27 and 30 sites.

\section{Keywords}

Finite Temperature Lanczos Method, Heisenberg Model, Kagome Lattice, Specific Heat

\section{Introduction}

Study about the spin-1/2 Heisenberg model on the kagome lattice [1]-[4] has remained to be formidable during several decades. Recently this model has been studied extensively to pursue a possible candidate for a quantum spin liquid [5]-[10]. Also as a spin-1/2 antiferromagnet with the kagome geometry, Kapellasite, $\mathrm{ZnCU}_{3}(\mathrm{OH})_{6} \mathrm{Cl}_{2}$ has been synthesized and has been studied about its magnetic properties [11]. These experimental works require theoretical studies on this system at finite temperature $T$. One of these studies is made by the high-temperature series expansion that has shown the quite stable results for the uniform susceptibility over the wide range of temperature [12]-[14]. But this study has failed to present conclusive results for the specific heat at low $T$. Reasons 
of this failure are that this method such as the high-temperature series expansion is difficult at low $T$, and that the specific heat of the model on the kagome lattice cannot be described by the power-law behavior at low $T$. Also we should realize that this method explains only a half of the entropy.

In this work, we will present calculations of the specific heat at low $T$ by the exact approach for small clusters. The exact approach is very powerful and extensively applied for study at zero $T$ [15]-[17], although the size is severely limited. There are few researches on the exact approach to calculations of the specific heat at finite $T$. Only some results on $L=18$ or $L=24$ clusters are found in [12] [13]. Here $L$ is the total number of sites. By these previous works, it is understood that ordinary calculations on $L=27$ or larger lattices are quite difficult because the system on the kagome lattice has the complicated structure at low $T$. We point out the Chebyshev polynomial expansion (CPE) as one method of the exact approaches at finite $T$ [18] [19]. Another method is the finite temperature Lanczos method (FTLM) [20] [21].While the polymial expansion method is well mathematically understood, in the FTLM we should be careful to control numerical errors. Due to this weakness there are a fewer applications of the FTLM, compared with the CPE [18], although both methods give us the same calculation costs.

However, recently the FTLM has been improved by making use of the full irreducible representation of the symmetry group [22], by applying the method to the microcanonical ensemble [23] or by combining it with the meanfield approach [24]. Also the improvement has been made by modifying the initial state for the Lanczos method [25]. As a result, we can make precise calculations of thermodynamic quantities of strongly correlated systems [22]. Also it has been applied to the Kondo lattice [26], and J1-J2 Heisenberg model [27].

Here we would like to emphasize an advantage of the FTLM that we calculate the specific heat at many values of $T$ without keeping states in the computer memory. This is quite important in study by limited resources of computers.

In calculations at low $T$, we need the large number $M$ of iterations for obtaining the orthogonal states in the FTLM. In calculations of $M>100$, we have to worry about errors on the orthogonality of the states. In order to keep that $M<100$, we propose a two-step method, which is some extension of the method, in which the initial state for the Lanczos method is modified [25].

In the first step, we use the CPE to generate a state $\exp \left(-\hat{H} / 2 T_{1}\right)|r\rangle$ at moderate $T_{1}$. Here $|r\rangle$ is a random vector. In the second step, we employ the FTLM. We call our proposed method as a two-step finite temperature Lanczos method (tFTLM).

In addition to this two-step strategy, we propose a sampling method for selecting the initial random state. It is well known that the random vector method is quite effective for the trace calculation of the partition function. If the Hamiltonian has some symmetry, the basis states are divided into symmetry sectors. In each symmetry sector we calculate the partition function. At the infinitely high $T$ a sampling weight of each symmetry sector is proportional to the number of basis states in the symmetry sector. But at very low $T$, the partition function depends extremely on the lowest energy $E_{s}$ of the symmetry sector $s$. Based on this consideration, we use a product of $\exp \left(-E_{s} / T_{1}\right)$ and the number of basis states for a sample weight of a sector.

Contents of this paper are as following. After a brief review on the FTLM, we propose our method in Section 2. In Section 3, we make an examination about our method in order to show that the tFTLM is effective at very low $T$. After the examination, we apply the tFTLM to calculate the specific heat of the spin- $1 / 2$ Heisenberg model on the kagome lattice. By results of $L=27$ and $L=30$ lattice we conclude that the specific heat shows the curve of the shoulder around $T=0.15$. A final section is devoted to a conclusion.

\section{Two-Step Finite Temperature Lanczos Method}

First we present a brief description on the FTLM. We consider calculations of a static expectation value of an operator $\hat{O}$ at $T$.

$$
\langle\hat{O}\rangle=\frac{1}{Z} \sum_{n}^{N}\left\langle n\left\langle\hat{O} \mathrm{e}^{-\beta \hat{H}}\right\rangle n\right\rangle, \quad Z=\sum_{n}^{N}\left\langle n\left\langle\mathrm{e}^{-\beta \hat{H}}\right\rangle n\right\rangle,
$$

with $\beta=1 / T$ and a sum over a complete orthonormal basis set $|n\rangle$. Here $N$ is the dimension of the vector space. Using the FTLM it is approximated by 


$$
\begin{gathered}
\langle\hat{O}\rangle \approx \frac{1}{Z} \sum_{s} \frac{N_{s}}{R} \sum_{r}^{R} \sum_{m}^{M} \mathrm{e}^{-\beta E_{m}^{(r)}}\left\langle r \mid \Psi_{m}^{(r)}\right\rangle\left\langle\Psi_{m}^{(r)}|\hat{O}| \mathrm{r}\right\rangle, \\
Z \approx \sum_{s} \frac{N_{s}}{R} \sum_{r}^{R} \sum_{m}^{M} \mathrm{e}^{-\beta E_{m}^{(r)}}\left|\left\langle r \mid \Psi_{m}^{(r)}\right\rangle\right|^{2},
\end{gathered}
$$

with a sum over symmetry sectors s of dimension $N_{s}$. The trace in (1) is replaced by the sum over $R^{\prime} \mathrm{s}$ random vectors $|r\rangle$ in (2) and (3) [28] [29]. $M$ is the number of iterations in the Lanczos procedure. On each random vector $|r\rangle$, the Lanczos procedure gives us the eigenenergies $E_{m}^{(r)}$ and their corresponding eigenvectors $\left|\Psi_{m}^{(r)}\right\rangle$, whose number is $M$. Because we adopt the random vector method, the calculation cost is of order of $R M$.

The accuracy of the FTLM depends on obtained eigenvalues in the Lanczos method. When a system size becomes large, we need a quite large number $M$ in order to obtain the eigenvalue that is equal or nearly equal to the lowest energy of the system. However, $M$ could not be large, because in the Lanczos method the error on the orthogonality becomes large as $M$ is large. The maximum value of $M$ is about 100 [18]. The limit of $M$ gives us the minimum $T$ where calculations are reliable.

This limitation can be avoided in the Lanczos method for the energy calculation at zero $T$ by repeating this method several times. In order to introduce this repetition into the FTLM, we propose a two-step method. In the first step we generate a state $\exp \left(-\beta_{1} \hat{H} / 2\right)|r\rangle$ at moderate $T_{1}=1 / \beta_{1}$.

$$
\begin{gathered}
\left|\phi_{r}\right\rangle=\mathrm{e}^{-\beta_{1} \hat{H} / 2}|r\rangle / Z_{1}(r)^{1 / 2}, \\
Z_{1}(r)=\left\langle r\left|\mathrm{e}^{-\beta_{1} \hat{H}}\right| r\right\rangle .
\end{gathered}
$$

For this calculations of $\left|\phi_{r}\right\rangle$, we use the CPE.

$$
\left|\phi_{r}\right\rangle \approx \sum_{k}^{K} c_{k}\left(\beta_{1}\right) T_{k}\left(\hat{H} / H_{0}\right)|r\rangle / Z_{1}(r)^{1 / 2} .
$$

Here $T_{k}(x)$ is the $k$-th Chebyshev polynomial. Here $H_{0}$ is determined in order that the absolute value of $\hat{H} / H_{0}$ is less than 1 .

The precision of the approximation in the first step calculation can be controlled by $K$. We would like to reduce the numerical error of the state $\left|\phi_{r}\right\rangle$ to $O\left(10^{-10}\right)$, so that $K$ is fixed by the value of $T_{1}$.

Next we carry out the second step procedure. Here we apply the Lanczos method, where we use this state $\left|\phi_{r}\right\rangle$ as the initial state.

$$
\begin{gathered}
\langle\hat{O}\rangle \approx \frac{1}{Z} \sum_{s} \frac{N_{s}}{R} \sum_{r}^{R} \sum_{m}^{M} \mathrm{e}^{-\left(\beta-\beta_{1}\right) E_{m}^{(r)}}\left\langle\phi_{r} \mid \Psi_{m}^{(r)}\right\rangle\left\langle\Psi_{m}^{(r)}|\hat{O}| \phi_{r}\right\rangle Z_{1}(r), \\
Z \approx \sum_{s} \frac{N_{s}}{R} \sum_{r}^{R} \sum_{m}^{M} \mathrm{e}^{-\left(\beta-\beta_{1}\right) E_{m}^{(r)}}\left|\left\langle\phi_{r} \mid \Psi_{m}^{(r)}\right\rangle\right|^{2} Z_{1}(r) .
\end{gathered}
$$

In the Lanczos procedure we obtain eigenenergies $E_{m}^{(r)}$ and their corresponding eigenvectors $\left|\Psi_{m}^{(r)}\right\rangle$, whose number is $M$.

Finally we describe a sampling method for selecting the initial random state. As described in the introduction, at low $T$ the partition function depends extremely on the lowest energy $E_{s}$ of the symmetry sector $s$. If $E_{s}$ differs from each other, it is not effective that only the number of basis states $N_{s}$ of the symmetry sector is used as the sampling weight. The Heisenberg model on the kagome is a typical example of cases that there are some differences between $E_{s}$ 's. For our calculations we use the product of $\exp \left(-E_{s} / T_{1}\right)$ and the number of basis states for the sample weight of a sector. Therefore a probability $P(s)$ of selecting $s$ is given by

$$
P(s)=\exp \left(-E_{s} / T_{1}\right) N_{s} / \sum_{s^{\prime}} \exp \left(-E_{s^{\prime}} / T_{1}\right) N_{s^{\prime}} .
$$

Using this probability $P(s)$, we obtain 


$$
Z \approx \sum_{r_{s}}^{R} P(s) \frac{1}{P(s)} \sum_{m}^{M} \mathrm{e}^{-\left(\beta-\beta_{1}\right) E_{m}^{\left(r_{s}\right)}}\left|\left\langle\phi_{r_{s}} \mid \Psi_{m}^{(r)}\right\rangle\right|^{2} Z_{1}\left(r_{s}\right),
$$

The following is a procedure for sampling. First we select $s$ according to the probability $P(s)$. Next we generate a random vector in the selected $s$. We apply the tFTLM to calculate the partition function. After that we multiply this partition function by the factor of $1 / P(s)$.

\section{The Specific Heat}

First we make an examination about our method by applying the tFTLM to calculations in the spin-1/2 Heisenberg model on the kagome lattice. The Hamiltonian of this model is defined by

$$
\hat{H}=\sum_{i, j} \vec{s}_{i} \cdot \vec{s}_{j} .
$$

Here $\vec{s}_{i}=\left(s_{i}^{x}, s_{i}^{y}, s_{i}^{z}\right)$ is a spin operator of the spin one-half at a site $i$. The sum is carried out over a pair of sites on a link of the kagome lattice.

For the examination of the tFTLM we calculate the specific heat on the kagome antiferromagnet of the $L=24$ lattice. Although the exact results on it cannot be available, the specific heat per site $C_{v}(T)$ must be quite small at extremely low $T$ on the finite lattice. In Figure 1, we plot the specific heat until $T=1.25 \times 10^{-3}$. The calculations are made using the FTLM and the tFTLM. The specific heat obtained by the FTLM of $M=60$ does not fall to the zero, but remains constant. Results by the FTLM of $M=80$ have the same tendency, though the constant value becomes small. About results by the tFTLM, the specific heat has fallen to the value that is consistent with zero. This figure shows that the tFTLM can be applied safely to the extremely low $T$.

Next we make calculations of the $C_{v}(T)$ of the spin-1/2 Heisenberg model on the kagome lattice. Here we give a description about parameters of the tFHTM on $L=27$ and $L=30$. In the first step we have two parameters of $T_{1}$ and $K . T_{1}=0.25$ is chosen in order to check our method, by comparing our results with results of the FTLM or of the high-temperature series expansion [12]. In order to reduce the calculation error into $10^{-10}$ about a given random vector, we need a ratio of coefficients $c_{K}\left(\beta_{1}\right) / c_{0}\left(\beta_{1}\right) \leq 10^{-10}$ in the Chebyshev polynomial (6). For obtaining this ratio, we determine $K=40$ in (6). In the second step the minimum value of $T$, at which calculations are made, is 0.0125 for making valuable discussions on the size dependence of the specific heat. For determining a value of $M$, we check the reliability of calculations using only one random vector. After this examination we fix that $M=80$.

Our results on clusters of $L=21,24,27,30$ are given in Figure 2. From the study by the high-temperature series expansion analysis [12] [13] we can say that the dependence of the $C_{v}(T)$ at $T=0.25$ or at the higher $T$ on the size is quite small. Our results support this little dependence on the cluster size. On data at $0.15<T<0.25$ we find the dependence of the specific heat $C_{v}(T)$ on the cluster size, but it is small. By this small dependence, we can say that the curve of the $C_{v}(T)$ on the temperature has changed from the curve at $T>0.25$. As the study of [12] [13] showed, the high-temperature series expansion could not explain this curve at this temperature. On the entropy $S_{e}\left(T_{0}\right)$ that is calculated by the integration from $T_{0}$ to the infinitely high $T$, we have that $S_{e}\left(T_{0}=0.25\right) \approx 0.5$. This small value suggests existence of the huge number of excited states, whose gap energies are small than $T=0.25$. At $T<0.15$, we can find the large dependence of the $C_{v}(T)$ on the cluster size. Therefore we could not mention a quantitative statement on the curve of the specific heat.

But at $0.04<T<0.15$ we can make a qualitative discussion. We find the qualitatively same results on data of $L=21$ and $L=27$, which show the shoulder of the $C_{v}(T)$ curve. The $C_{v}(T)$ on $L=24$ shows the clear peak. This fact gives us the qualitative difference between the $C_{v}(T)$ on the even size lattice and that of the odd size lattice. On the $L=30$ lattice, the peak of the $C_{v}(T)$ curve is not found, and only the shoulder is seen. This tendency suggests that the curve of the $C_{v}(T)$ on the larger even size lattice will agree with the curve on $L=27$. Summarizing our calculations, they show that this shoulder survives at the large $L$ limit at $0.04<T<0.25$.

\section{Conclusions}

In this work, we presented two improvements on the FTLM in order to apply this method to systems at very low 


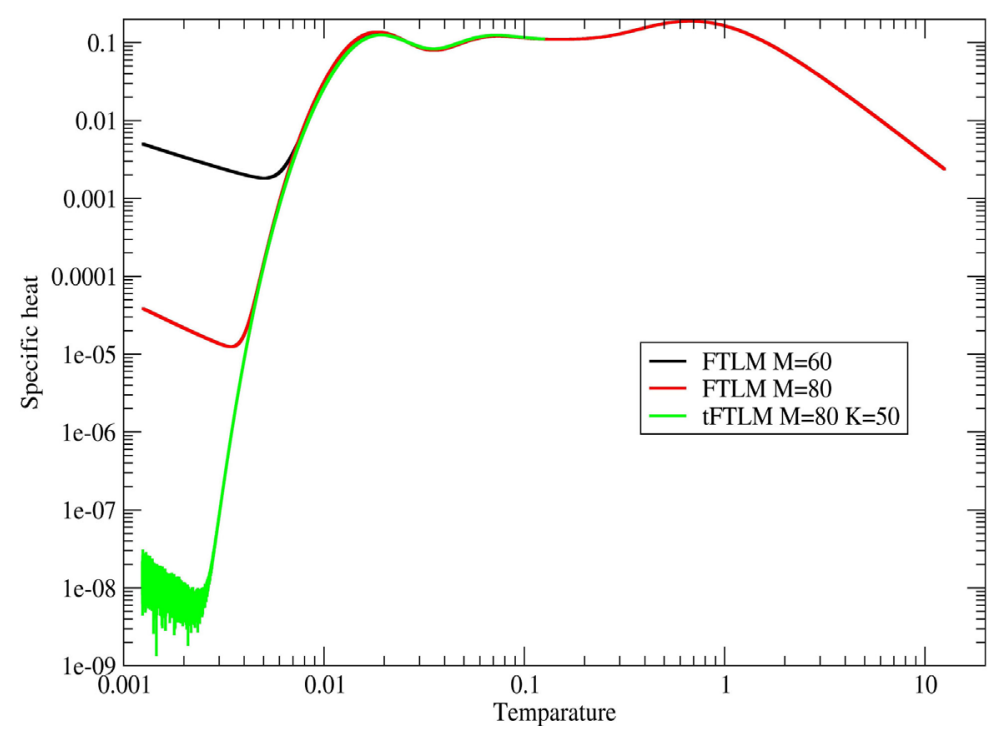

Figure 1. The specific heat at low temperature on the kagome lattice of $L=24$ In the tFTHM, $T_{1}=0.25$. The number of sampling random vectors is 1000 .

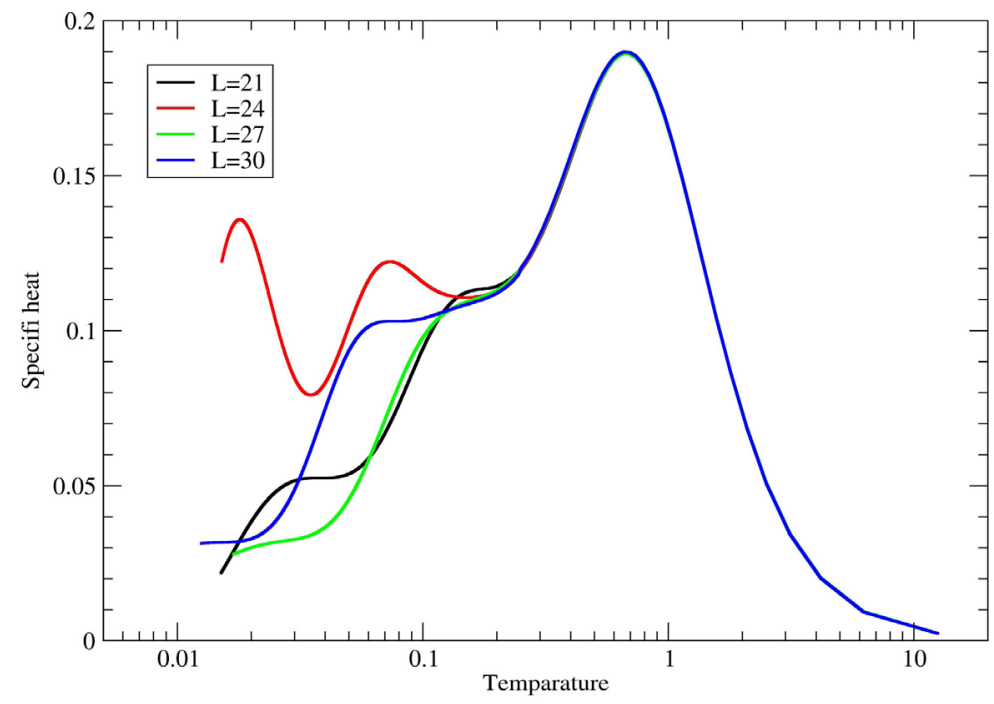

Figure 2. The specific heat on the kagome lattice of $L=21,24,27,30$. On calculations of $L=30$ and $L=27$, parameters of $T_{1}=0.25, K=40$ and $M=80$ are adopted. The number of sampling random vectors is 1000 . The statistical error is less than $3 \%$, so that it is not shown.

temperature. One improvement was to introduce the two-step FTLM, tFTLM. In the first step we used the Chebyshev polynomial expansion to calculate $\exp \left(-\beta_{1} \hat{H} / 2\right)|r\rangle$ at moderate temperature $T_{1}=1 / \beta_{1}$. In the second step, we applied the FTLM using the calculated state as the initial state. By this two-step method, we don't have to worry about the error of the Lanczos method. The following is a reason for this improvement. We need a large number of iterations on the Lanczos method at low temperature. This number is limited by the numerical error. While in the Chebyshev polynomial expansion this error is under the control. Therefore the numerical error can be quite small at the first step calculation. By the first step calculation we can reduce the number of the iteration of the Lanczos method in the second calculation. 
Another improvement was to employ the effective sampling method for selecting a random vector. Using this sampling we can improve the efficiency of calculations. By the tFTLM, we calculated the specific heat of the spin-1/2 Heisenberg model on the kagome lattice of 30 sites. Our results confirmed the little dependence of the specific heat on the cluster size at $T>0.25$. At $0.15<T<0.25$ the dependence on the cluster size was small, so that we concluded that the curve of the specific heat changed from the curve at the higher temperature. Also our calculation suggested that the shoulder of the curve survived at the large cluster size at $0.04<T<0.3$. Summarizing this work, we proposed the tFTLM that was quite effective for numerical study at extremely low temperature and we presented definite results about the specific heat of the Heisenberg model on the $L=30$ kagome lattice.

We would like to make comments on further applications of the method. In this paper we applied the tFTLM to only static quantities at finite $T$. But it is easy to apply our method to the dynamical quantities. In study of the spin liquid model on the kagome, the work [7] has discussed the dynamical susceptibility that can be observed by neutron scattering and NMR. Also in numerical studies this is an issue to be examined, if we realize that around $T=0.1$ results of the specific heat on $L=30$ differ from results on $L=24$, while the uniform susceptibility has showed the little dependence. Therefore it is very interesting to examine a size dependence of the dynamical susceptibility.

Also we comment on implication of our results of the specific heat. They require the more careful study on the spin liquid model, which suggests the power law behavior of the specific heat at low $T$. The size dependence has become small around $T=0.1$ in our results. Therefore we need a consistent description about our results and about the power-law behavior of the specific heat. But this is not easy as discussed in [12]. Our study suggests that the unified understanding of the specific heat is one of important issues on the Heisenberg model on the kagome lattice.

\section{Acknowledgements}

M. T. thanks Dr. Yasuko Munehisa for every encouragement on my study.

\section{References}

[1] Zeng, C. and Elser, V. (1990) Numerical Studies of Antiferromagnetism on a Kagome Net. Physical Review B, 42, 8436-8444. http://dx.doi.org/10.1103/PhysRevB.42.8436

[2] Elstner, N. and Young, A.P. (1994) Spin-1/2 Heisenberg Antiferromagnet on the Kagome Lattice: High-Temperature Expansion and Exact-Diagonalization Studies. Physical Review B, 50, 6871-6876. http://dx.doi.org/10.1103/PhysRevB.50.6871

[3] Nakamura, T. and Miyashita, S. (1995) Thermodynamic Properties of the Quantum Heisenberg Antiferromagnet on the Kagome Lattice. Physical Review B, 52, 9174-9177. http://dx.doi.org/10.1103/PhysRevB.52.9174

[4] Lecheminant, P., Bernu, B., Lhuillier, C., Pierre, L. and Sindzingre, P. (1997) Order versus Disorder in the Quantum Heisenberg Antiferromagnet on the Kagome Lattice Using Exact Spectra Analysis. Physical Review B, 56, 2521-2529. http://dx.doi.org/10.1103/PhysRevB.56.2521

[5] Depenbrock, S., McCulloch, I.P. and Schollwoeck, U. (2012) Nature of the Spin-Liquid Ground State of the S = 1/2 Heisenberg Model on the Kagome Lattice. Physical Review Letter, 109, 067201-067201-6. http://dx.doi.org/10.1103/PhysRevLett.109.067201

[6] Misguich, G., Serban, D. and Pasquier, V. (2002) Quantum Dimer Model on the Kagome Lattice: Solvable DimerLiquid and Ising Gauge Theory. Physical Review Letter, 89, 137202-137202-4. http://dx.doi.org/10.1103/PhysRevLett.89.137202

[7] Hermele, M., Ran, Y., Lee, P.A. and Wen, X.G. (2008) Properties of an Algebraic Spin Liquid on the Kagome Lattice. Physical Review B, 77, 224413-224413-23. http://dx.doi.org/10.1103/PhysRevB.77.224413

[8] Iqbal, Y., Becca, F., Sorella, S. and Poilblanc, D. (2013) Gapless Spin-Liquid Phase in the Kagome Spin-1/2 Heisenberg Antiferromagnet. Physical Review B, 87, 060405-060405-5. http://dx.doi.org/10.1103/PhysRevB.87.060405

[9] Messio, L., Bernu, B. and Lhuillier, C. (2012) Kagome Antiferromagnet: A Chiral Topological Spin Liquid? Physical Review Letter, 108, 207204-207204-5. http://dx.doi.org/10.1103/PhysRevLett.108.207204

[10] Clark, B.K., Kinder, J.M. Neuscamman, E., Chan, K.C. and Lawler, M.J. (2013) Striped Spin Liquid Crystal Ground State Instability of Kagome Antiferromagnets. Physical Review Letter, 111, 187205-87205-5.

[11] Fak, B., Kermarrec, E., Messio, L., Bernu, B., Lhuillier, C., Bert, F., Mendels, P., Koteswararao, B., Bouquet, F., Ol- 
livier, J., Hillier, A.D., Amato, A., Colman, R.H. and Wills, A.S. (2012) Kapellasite: A Kagome Quantum Spin Liquid with Competing Interactions Experiments. Physical Review Letter, 109, 037208-037208-5.

http://dx.doi.org/10.1103/PhysRevLett.109.037208

[12] Misguich, G. and Bernu, B. (2005) Specific Heat of the $S=1 / 2$ Heisenberg Model on the Kagome Lattice: HighTemperature Series Expansion Analysis. Physical Review B, 71, 014417-014417-7. http://dx.doi.org/10.1103/PhysRevB.71.014417

[13] Misguich, G. and Sindzingre, P. (2007) Magnetic Susceptibility and Specific Heat of the Spin-1/2 Heisenberg Model on the Kagome Lattice and Experimental Data on $\mathrm{ZnCu}_{3}(\mathrm{OH})_{6} \mathrm{Cl}_{2}$. The European Physical Journal B, 59, 305-309. http://dx.doi.org/10.1140/epjb/e2007-00301-6

[14] Singh, R.R.P. and Oitmaa, J. (2012) High-Temperature Series Expansion Study of the Heisenberg Antiferromagnet on the Hyperkagome Lattice: Comparison with $\mathrm{Na}_{4} \mathrm{Ir}_{3} \mathrm{O}_{8}$. Physical Review B, 85, 104406-104406-4. http://dx.doi.org/10.1103/PhysRevB.85.104406

[15] Laeuchli, A.M., Sudan, J. and Sorensen, E.S. (2011) Ground-State Energy and Spin Gap of Spin-12 Kagome-Heisenberg Antiferromagnetic Clusters: Large-Scale Exact Diagonalization Results. Physical Review B, 83, 212401-212404. http://dx.doi.org/10.1103/PhysRevB.83.212401

[16] Nakano, H. and Sakai, T. (2011) Numerical-Diagonalization Study of Spin Gap Issue of the Kagome Lattice Heisenberg Antiferromagnet. Journal of the Physical Society of Japan, 80, 053704-053708. http://dx.doi.org/10.1143/JPSJ.80.053704

[17] Isoda, M., Nakano, H. and Sakai, T. (2011) Specific Heat and Magnetic Susceptibility of Ising-Like Anisotropic Heisenberg Model on Kagome Lattice. Journal of the Physical Society of Japan, 80, 084704-084706. http://dx.doi.org/10.1143/JPSJ.80.084704

[18] Weise, A., Wellein, G., Alvermann, A. and Fehske, H. (2006) The Kernel Polynomial Method. Review Modern Physics, 78, 275-306. http://dx.doi.org/10.1103/RevModPhys.78.275

[19] Iitaka, T., Nomura, S., Hirayama, H., Zhao, X., Aoyagi, Y. and Sugano, T. (1997) Calculating the Linear Response Functions of Noninteracting Electrons with a Time-Dependent Schroedinger Equation. Physical Review E, 56, $1222-$ 1229. http://dx.doi.org/10.1103/PhysRevE.56.1222

[20] Jaklic, J. and Prelpvsek, P. (1994) Lanczos Method for the Calculation of Finite-Temperature Quantities in Correlated Systems. Physical Review B, 49, 5065-5068. http://dx.doi.org/10.1103/PhysRevB.49.5065

[21] Jaklic, J. and Prelpvsek, P. (2000) Finite-Temperature Properties of Doped Antiferromagnets. Advance Physics, 49, 1-92. http://dx.doi.org/10.1080/000187300243381

[22] Schnack, J. and Wendland, O. (2010) Properties of Highly Frustrated Magnetic Molecules Studied by the Finite-Temperature Lanczos Method. The European Physical Journal B, 78, 535-541. http://dx.doi.org/10.1140/epjb/e2010-10713-8

[23] Long, M.W., Prelovsek, P., El Shawish, S., Karadamoglou, J. and Zotos, X. (2003) Finite-Temperature Dynamical Correlations Using the Microcanonical Ensemble and the Lanczos Algorithm. Physical Review B, 68, 235106235106-10. http://dx.doi.org/10.1103/PhysRevB.68.235106

[24] Capone, M., de’Medici, L. and Georges, A. (2007) Solving the Dynamical Mean-Field Theory at Very Low Temperatures Using the Lanczos Exact Diagonalization. Physical Review B, 76, 245116-245116-6. http://dx.doi.org/10.1103/PhysRevB.76.245116

[25] Aichhorn, M., Daghofer, M., Evertz, H.G. and von der Linden, W. (2003) Low-Temperature Lanczos Method for Strongly Correlated Systems. Physical Review B, 67, 161103-161103-4. http://dx.doi.org/10.1103/PhysRevB.67.161103

[26] Zerec, I., Schmidt, B. and Thalmeier, P. (2006) Kondo Lattice Model Studied with the Finite Temperature Lanczos Method. Physical Review B, 73, 245108-245108-6. http://dx.doi.org/10.1103/PhysRevB.73.245108

[27] Schmidt, B., Thalmeier, P. and Shannon, N. (2007) Magnetocaloric Effect in the Frustrated Square Lattice J1-J2 Model. Physical Review B, 76, 125113-125113-19. http://dx.doi.org/10.1103/PhysRevB.76.125113

[28] Hams, A. and De Raedt, H. (2000) Fast Algorithm for Finding the Eigenvalue Distribution of Very Large Matrices. Physical Review E, 62, 4365-4377. http://dx.doi.org/10.1103/PhysRevE.62.4365

[29] Iitaka, T. and Ebisuzaki, T. (2004) Random Phase Vector for Calculating the Trace of a Large Matrix. Physical Review E, 69, 057701-057701-4. http://dx.doi.org/10.1103/PhysRevE.69.057701 
Scientific Research Publishing (SCIRP) is one of the largest Open Access journal publishers. It is currently publishing more than 200 open access, online, peer-reviewed journals covering a wide range of academic disciplines. SCIRP serves the worldwide academic communities and contributes to the progress and application of science with its publication.

Other selected journals from SCIRP are listed as below. Submit your manuscript to us via either submit@scirp.org or Online Submission Portal.
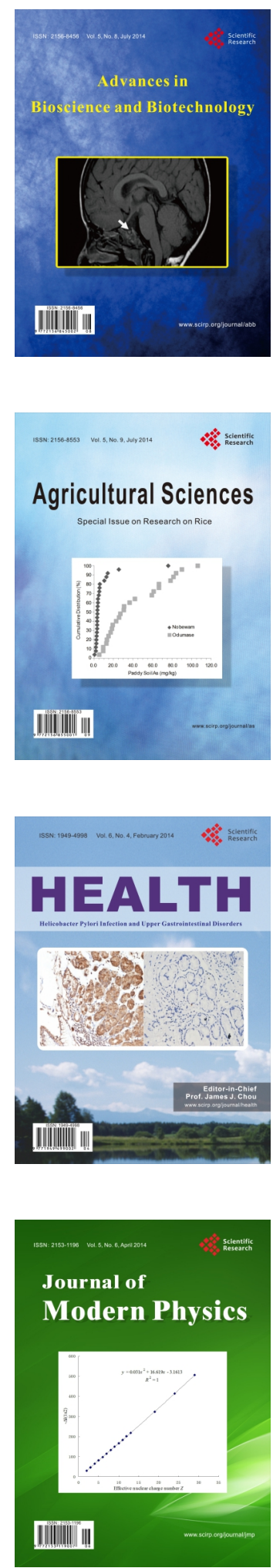
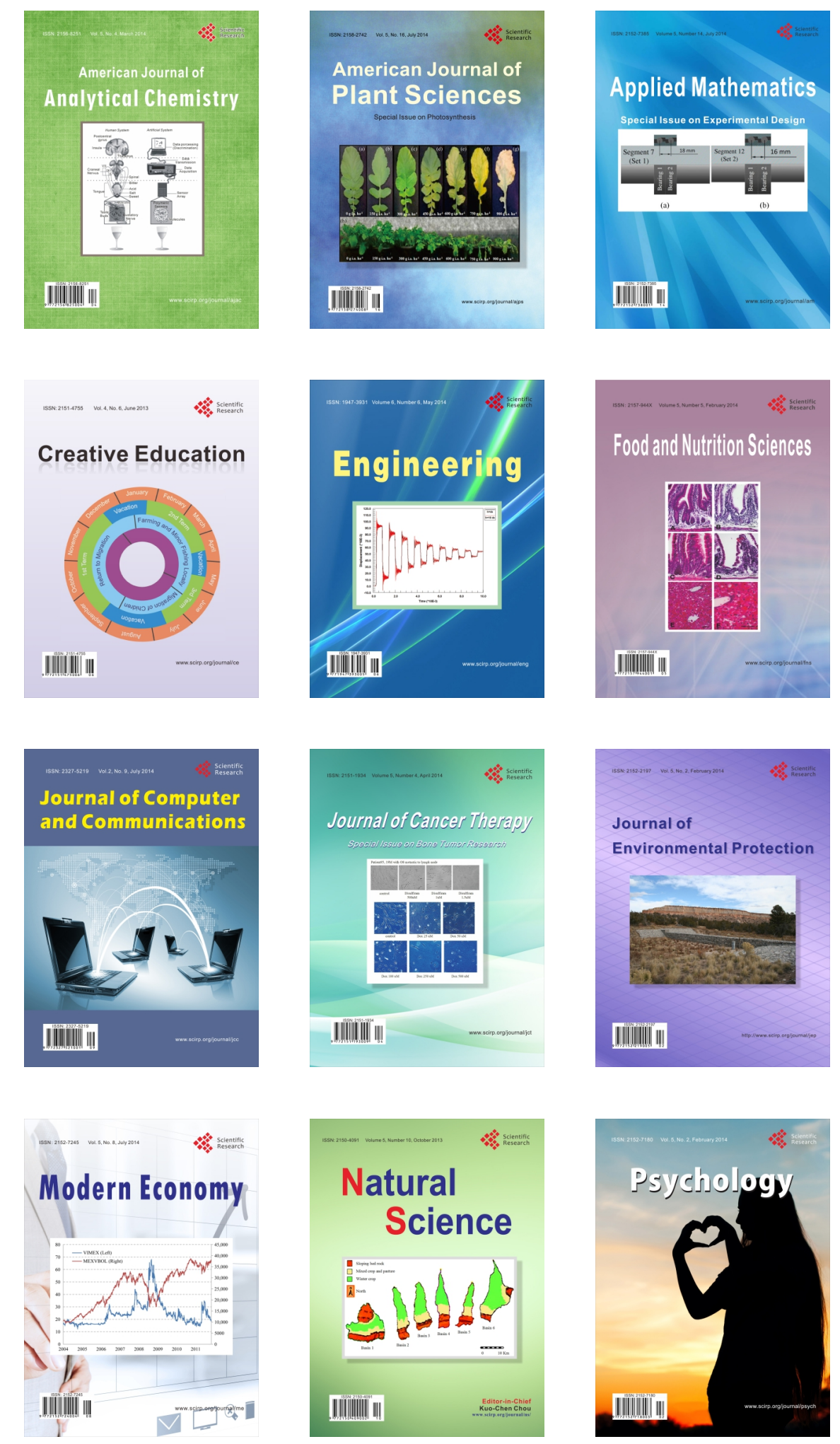